\title{
Subnational estimates of health in low- and middle-income countries: a geospatial approach with interpolation and raster analysis
}

\author{
Lois Park ${ }^{\mathrm{a}, *}$ \\ ${ }^{a}$ Spatial Sciences Institute, University of Southern California, LoisPark@usc.edu \\ * Corresponding author
}

\begin{abstract}
:
Program and policy planners are eager for population health data at subnational levels in the era of the Sustainable Development Goals (SDGs). The Demographic and Health Survey (DHS) is a major source of data for tracking population health in low- and middle-income countries, with over 300 surveys in 90 countries since the 1980s. The DHS is usually nationally representative and commonly sampled to be representative at one administrative level below the national level, often the region. However, regional estimates are still too broad and general to meet the needs of decision-makers, who desire to make data-driven decisions at more local levels. In addition to data on health the DHS collects and publishes the coordinates of all sampled clusters. This analysis proposes and demonstrates a spatial approach to generating subnational estimates of health using the publicly available spatial data. By pairing kriging for interpolation with raster analysis for weighting by population size and aggregation, estimates of antenatal care coverage are generated at the district level in Malawi, using data from the 2015-16 DHS. Preliminary results show that estimates generated with this geospatial approach are well-aligned with estimates published by the DHS. The resulting maps with a continuous surface of estimates is also able to demonstrate subnational heterogeneity in health status in a way standard choropleth maps are unable. While the approach requires refinement and further validation, it is a creative way to use spatial analysis tools to address the demand for health indicators at local levels.
\end{abstract}

Keywords: global health, demographic and health survey, LMIC, SDG, geospatial, interpolation, kriging 